\title{
Avoiding Unnecessary Pulmonary CT Angiography by Using a Combination of Clinical Criteria and D-Dimer Thresholds
}

\author{
Lana Hirai Gimber ${ }^{1}$, Jamie Leong ${ }^{2}$, Lauren Todoki ${ }^{2}$, Hyo-Chun Yoon ${ }^{2}$ \\ ${ }^{1}$ Department of Medical Imaging, University of Arizona, Tucson, USA \\ ${ }^{2}$ Department of Diagnostic Imaging, Kaiser Foundation Hospital, Los Angeles, USA \\ Email: lgimber@email.arizona.edu
}

Received March 18, 2013; revised April 20, 2013; accepted April 27, 2013

Copyright (C) 2013 Lana Hirai Gimber et al. This is an open access article distributed under the Creative Commons Attribution License, which permits unrestricted use, distribution, and reproduction in any medium, provided the original work is properly cited.

\begin{abstract}
Objective: To evaluate what specific combination of clinical criteria and d-dimer values may yield at least a $10 \%$ positive pulmonary embolism (PE) rate in patients undergoing pulmonary CT angiography (CTA). Materials and Methods: Retrospective review of all patients presenting to the Emergency Department with possible PE who underwent pulmonary CTA and had a d-dimer drawn. Wells scores were retrospectively assigned based on data gathered through medical records. Results: During a 29-month period, 1110 patients underwent pulmonary CTA. Of these, 773 also had a d-dimer drawn. These subjects were stratified based on serum d-dimer levels into negative $(\leq 4 \mu \mathrm{g} / \mathrm{ml})$, nonpositive $(0.41-1.0 \mu \mathrm{g} / \mathrm{ml})$, or positive $(>1.0 \mu \mathrm{g} / \mathrm{ml})$ d-dimer categories. The prevalence of positive CTA studies was $>10 \%$ only in the positive d-dimer group. Subjects were also stratified based on their Wells score into three clinical categories: low $($ score $<2$ ), intermediate (score $=2-6$ ), and high risk of pulmonary embolism (score $>6$ ). The prevalence of positive CTA was $>10 \%$ only in the group of subjects with high clinical risk. When stratified according to both Wells criteria and d-dimer, only those patients with intermediate or high clinical risk combined with a positive d-dimer $(>1.0 \mu \mathrm{g} / \mathrm{ml})$ had a prevalence of positive pulmonary CTA $>10 \%$. By limiting the use of CTA studies to those patients with positive d-dimer values or high clinical risk, $438(55.4 \%)$ patients could have avoided CTA imaging. Conclusion: Utilizing CTA only in patients suspected of PE with a combination of high clinical risk based on a Wells criteria threshold score $>6$ and a serum d-dimer cutoff of $1 \mu \mathrm{g} / \mathrm{ml}$ would increase the prevalence of positive pulmonary CTA studies above $10 \%$ and avoid a large number of CTA imaging studies.
\end{abstract}

Keywords: Pulmonary CT Angiography; Pulmonary Embolism; Wells Scores; D-Dimer Values

\section{Introduction}

The evaluation and management of patients suspected of acute pulmonary embolism (PE) in the emergency department setting is evolving. The ready availability of multidetector row CT scanners and quantitative serum d-dimer assays with rapid turnaround times have changed physician attitudes toward PE. It seems that physicians are much more likely to entertain the diagnosis of acute $\mathrm{PE}$ in their patients and to order tests accordingly. However, with the increasing ease of diagnosis, it is becoming clear that pulmonary CT angiography (CTA) is over utilized $[1,2]$. Studies have yet to demonstrate that this increased utilization of pulmonary CTA significantly decreases patient morbidity or mortality associated with PE, a disease whose incidence, prevalence and natural history is poorly understood [3]. In fact, the opposite may be true. Pulmonary CT has its own risks from exposure to ionizing radiation $[4,5]$ and contrast-induced nephropathy [6]. Furthermore, not all PE may be clinically relevant $[3,7]$.

The use of a clinical decision rule and quantitative serum d-dimer assay can obviate the need for pulmonary CTA in many patients $[8,9]$.

A recent study by Costantino et al. suggests that for pulmonary CTA to be considered a diagnostic imaging tool, there should be at least a $10 \%$ prevalence rate of positive PE among those patients undergoing CTA [1]. Hui et al. compared the prevalence of PE in CTA studies versus the prevalence of coincidental PE in patients undergoing contrast-enhanced CT scans for other indications and noted a prevalence of $11.8 \%$ in those being evaluated specifically for PE in the CTA group compared 
to a prevalence of $1.8 \%$ in those patients in whom PE was not suspected [10]. Two additional recent studies reported similar prevalence of PE in patients undergoing CT for other indications that varied depending on comorbid conditions, but in general hovered around $2 \%$ $[11,12]$. Therefore, it appears that a PE prevalence of $2 \%$ or less in a cohort of patients undergoing CTA would indicate overutilization. The purpose of this study was to determine which combination of clinical criteria and d-dimer values would allow at least a $10 \%$ CTA positive rate in patients being evaluated in an emergency medical department for acute PE and to avoid CTA in patient groups in whom the prevalence of PE would be $2 \%$ or less.

\section{Methods}

Institutional review board approval with a waiver of consent was obtained for a retrospective review of all subjects seen in an urgent care/ emergency room setting with a possible diagnosis of acute PE who underwent pulmonary CTA. All patients were members of an HMO where all care is provided within the network. A common electronic medical record allowed access to all patient data, which was necessary to find approximately 1000 patients for analysis. We reviewed the electronic medical record to determine patient age, gender, d-dimer result, if any, and pulmonary CTA result.

In the subset of these patients who also had a serum d-dimer level drawn, one observer reviewed all the clinical information in the electronic medical record associated with the emergency department encounter leading to the pulmonary CTA. Wells scores for these patients were retrospectively assigned. The Wells criteria have been validated as a method to stratify a patient's clinical probability of PE [8]. Points were assigned for each of the following clinical signs or symptoms: PE as likely or more likely than any alternative diagnosis, 3.0 points; signs or symptoms of deep venous thrombosis (leg swelling or painful palpation in the region of a deep vein), 3 points; heart rate higher than 100 beats/min, 1.5 points; immobilization (bedrest for 3 consecutive days) or surgery within past 4 weeks, 1.5 points; previous diagnosis of PE or DVT, 1.5 points; hemoptysis, 1.0 points; active malignancy (within past 6 months), 1.0 points. Most of these clinical signs and symptoms could be unambiguously determined from the electronic medical record documenting the emergency department encounter.

For the first variable, which is subject to individual interpretation, we used the following algorithm to determine if PE was as likely or more likely than any alternative diagnosis: If the patient's chief complaint on record was shortness of breath or dyspnea, then we assumed PE was the most likely diagnosis unless 1) the patient had a history of congestive heart failure and chest $\mathrm{x}$-ray was suggestive of edema or 2) the patient had signs and symptoms of a respiratory infection and an abnormal chest x-ray or 3) the patient had a history of asthma and clinical symptoms of an asthma exacerbation. If the patient' chief complaint was chest pain, then we assumed PE was the most likely diagnosis unless the patient had a history of coronary artery disease, prior myocardial infarction or cardiomyopathy. However, if the chest pain was further described as substernal, crushing or radiating to the back or left arm, PE was not assumed to be the most likely diagnosis. For a chief complaint of unilateral leg pain or swelling, PE was assumed the most likely diagnosis unless there was a specific finding in the reported history to suggest a more likely alternative diagnosis. Finally, if the emergency physician commented on a high likelihood of PE anywhere in the visit record, then we assumed that PE was the most likely diagnosis irrespective of the chief complaint.

The original studies utilizing Wells criteria segregated patients into three clinical risk strata for pulmonary embolism based on their score: low (score $<2$ ); intermediate (score 2 - 6) and high clinical risk for pulmonary embolism (score $>6$ ) [8]. However, a more recent study has validated a dichotomous segregation of patients based on their Wells score into those who are unlikely to have PE and those likely to have PE [9]. We segregated our subjects based on their Wells score using the 3 clinical risk strata and then repeated the analysis using the dichotomous strata limited to low clinical probability of PE (score $<4)$ and high clinical probability of PE (score $>$ 4).

In each analysis, the subjects were also segregated based on their serum d-dimer levels into those with negative serum d-dimer $(\leq 4 \mu \mathrm{g} / \mathrm{ml})$, nonpositive serum $\mathrm{d}$ dimer $(0.41-1.0 \mu \mathrm{g} / \mathrm{ml})$, or positive d-dimer $(>1.0 \mu \mathrm{g} / \mathrm{ml})$ using the latex agglutination technique (STA D-DI; DiagnosticaStago, Parsippany, NJ). We evaluated the conditions under which at least $10 \%$ of subjects undergoing pulmonary CTA would have a positive finding of acute PE. Finally, we evaluated which combinations of clinical risk stratification and d-dimer values resulted in cohorts of patients in whom the prevalence of PE was $\leq 2 \%$.All statistical analysis was performed using STATA v 7.0 (Stata, Inc, College Station, Tx).

\section{Results}

During the 29 months from January 2007 through May 2009, 1110 patients seen by the Emergency Medicine Department with suspected PE underwent pulmonary CTA. Sixty-three (5.7\%) had the diagnosis of PE based on CTA interpretation. Almost $70 \%(\mathrm{n}=773)$ of these patients had a serum d-dimer drawn at the time of pulmonary CTA. There was no significant difference in the 
prevalence of PE $(n=16)$ in the 337 patients who did not have a d-dimer drawn versus the prevalence of PE $(n=$ 47 ) in the patients who had a serum d-dimer (continuity corrected $\chi^{2}=0.78 ; p=0.38$ ).

The 773 patients with serum d-dimer levels were divided into three groups based on these d-dimer levels: Negative $(\leq 0.4 \mu \mathrm{g} / \mathrm{ml})$, nonpositive $(0.41-1.0 \mu \mathrm{g} / \mathrm{ml})$, or positive $(>1.0 \mu \mathrm{g} / \mathrm{ml})$. Although the latex agglutination technique we used in the study (STA D-DI; Diagnostica Stago, Parsippany, NJ) states a negative result as $\leq 0.4$ $\mu \mathrm{g} / \mathrm{ml}$, for the purposes of our study we designated anything $\leq 1.0 \mu \mathrm{g} / \mathrm{ml}$ as a nonpositive result. Therefore, only a level $>1.0 \mu \mathrm{g} / \mathrm{ml}$ was considered positive in our study. These ranges were chosen because previous studies using these thresholds have demonstrated a very low prevalence of acute $\mathrm{PE}$ in patients with serum d-dimer levels $\leq$ $1.0 \mu \mathrm{g} / \mathrm{ml}[13,14]$. The pulmonary CTA results based on these d-dimer categories are shown in Table 1. There was no significant difference in the prevalence of positive CTA studies for subjects with a negative d-dimer value and those with a nonpositive d-dimer value (Fisher exact $p=0.598$ ). However, there was a significant difference in the prevalence of positive pulmonary CTA studies for each pairwise comparison between the positive d-dimer group and the negative or nonpositive d-dimer groups (continuity corrected $\chi^{2}=9.63 ; \mathrm{p}=0.002$ and $\chi^{2}=33.90 ; \mathrm{p}<0.0001$, respectively). The prevalence of pulmonary CTA studies was $>10 \%$ only in subjects in the positive d-dimer group which was $12.4 \%$.

The same 773 subjects were first stratified into three groups based on their clinical risk for PE using the Wells clinical decision rule: low clinical risk (score $<2$ ), intermediate risk (score $2-6$ ), and high risk (score $>6$ ). The prevalence of each clinical criterion of the Wells score is presented in Table 2. The high prevalence of the first criterion stems from our liberal interpretation of the likelihood of PE based on the chief complaint.

The pulmonary CTA results based on these clinical risk categories are shown in Table 3. There was no significant difference in the prevalence of positive CTA studies for subjects with low clinical risk when compared with those with intermediate risk (continuity corrected $\chi^{2}$ $=3.22 ; \mathrm{p}=0.108)$. Significant differences were found in

Table 1. Positive pulmonary CTA results based on d-dimer categories.

\begin{tabular}{cccc}
\hline & Pos PE & Neg/Ind PE & Total \\
\hline Neg D-dimer $(\leq 0.4 \mu \mathrm{g} / \mathrm{ml})$ & $0(0 \%)$ & $70(100.0 \%)$ & 70 \\
Nonpos D-dimer $(0.41-1.0 \mu \mathrm{g} / \mathrm{ml})$ & $5(1.4 \%)$ & $358(98.6 \%)$ & 363 \\
Pos D-dimer $(>1.0 \mu \mathrm{g} / \mathrm{ml})$ & $42(12.4 \%)$ & $298(87.6 \%)$ & 340 \\
Total & $47(6.1 \%)$ & $726(93.9 \%)$ & 773 \\
\hline
\end{tabular}

Table 2. Wells criteria used to determine risk for PE.

\begin{tabular}{ccc}
\hline Criteria & N & $\%$ \\
\hline PE more likely than alternative diagnosis & 519 & 67.1 \\
Signs/symptoms of DVT & 64 & 8.3 \\
History of VTE & 34 & 4.4 \\
Tachycardia (HR $>100)$ & 209 & 27.0 \\
Recent $(<4$ wks) surgery or immobilization & 44 & 5.7 \\
Hemoptysis & 18 & 2.3 \\
Active Cancer & 72 & 9.3 \\
\hline
\end{tabular}

Table 3. Positive pulmonary CTA results based on Wells clinical decision rule.

\begin{tabular}{|c|c|c|c|}
\hline & Pos PE & Neg/Ind PE & Total \\
\hline Low risk $(<2)$ & $6(2.6 \%)$ & $223(97.4 \%)$ & 229 \\
\hline Intermediate risk $(2-6)$ & $29(5.6 \%)$ & $485(94.4 \%)$ & 514 \\
\hline High risk $(>6)$ & $12(40 \%)$ & $18(60 \%)$ & 30 \\
\hline Total & $47(6.1 \%)$ & $726(93.9 \%)$ & 773 \\
\hline
\end{tabular}

the prevalence of positive pulmonary CTA studies for each of the pairwise comparisons between the high clinical risk group versus the low or intermediate risk groups (Fisher exact $\mathrm{p}<0.001$ for both comparisons). A prevalence of positive CTA $>10 \%$ was found only in the group of subjects with high clinical risk in whom the prevalence of positive PE by CTA was $40 \%$.

As shown in Table 4, when the 773 patients were stratified by both their d-dimer values and clinical risk, only those patients with positive d-dimer who also had intermediate or high clinical risk had a prevalence of positive pulmonary CTA greater than $10 \%$. For all other groups of patients, the prevalence of positive pulmonary CTA studies was less than $5 \%$. Patients in the groups with negative or nonpositive $\mathrm{d}$-dimer did not have a prevalence of positive PE by CT of $>2 \%$ and would not likely benefit from CTA irrespective of the clinical risk.

The Christopher study utilized a Wells score $>4$ as a clinically useful threshold to separate patients who are unlikely or likely to have acute PE [9]. Therefore, we also stratified our patients using the same scoring scheme: low risk (score $\leq 4)$ versus high risk (score $>4)$. As expected, there was a significant difference in the prevalence of positive CTA between the two groups (continuity correct $\chi^{2}=22.26 ; \mathrm{p}<0.001$ ). The data in Table 5 demonstrate that among the 547 patients with low risk (score $<4$ ), only19 $(3.5 \%)$ proved to have PE by CTA. There were $28(12.4 \%)$ cases of positive PE by CTA among the 226 patients with high clinical risk (score $>4$ ). Only patients with both a positive d-dimer level with 
Table 4. Prevalence of positive pulmonary CTA stratified according to d-dimer and Wells criteria categories.

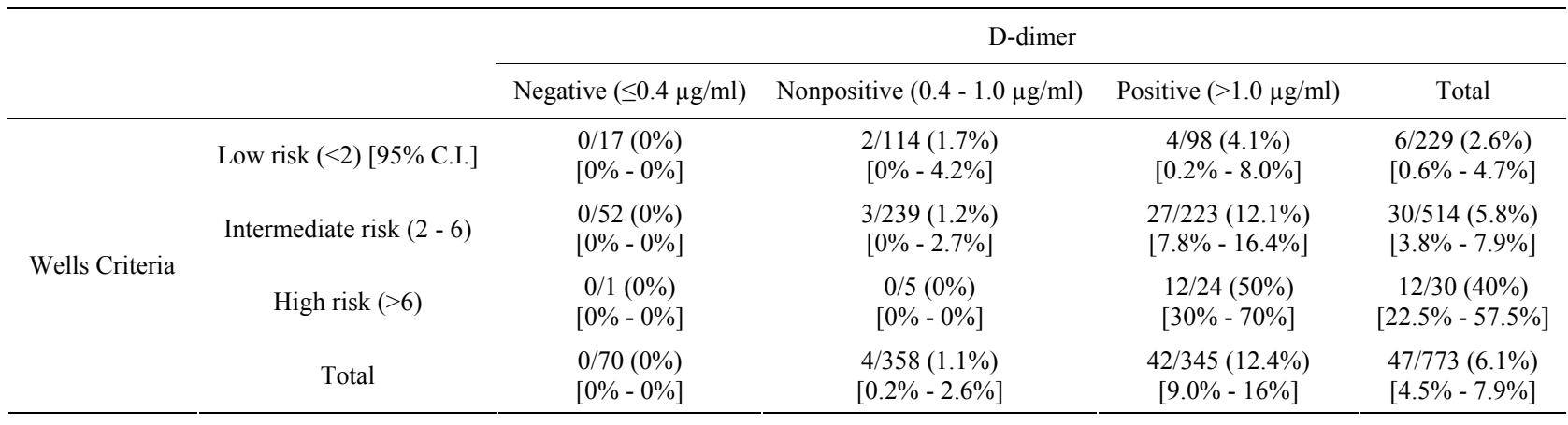

Table 5. Prevalence of positive pulmonary CTA stratified according to d-dimer and dichotomous Wells criteria categories.

\begin{tabular}{|c|c|c|c|c|c|}
\hline & & \multicolumn{4}{|c|}{ D-dimer } \\
\hline & & Negative $(\leq 0.4 \mu \mathrm{g} / \mathrm{ml})$ & Nonpositive $(0.4-1.0 \mu \mathrm{g} / \mathrm{ml})$ & Positive $(>1.0 \mu \mathrm{g} / \mathrm{ml})$ & Total \\
\hline \multirow{3}{*}{ Wells Criteria } & Low risk $(\leq 4)[95 \%$ C.I. $]$ & $\begin{array}{l}0 / 51(0 \%) \\
{[0 \%-0 \%]}\end{array}$ & $\begin{array}{l}2 / 278(0.7 \%) \\
{[0 \%-1.7 \%]}\end{array}$ & $\begin{array}{c}17 / 218(7.8 \%) \\
{[4.3 \%-11.4 \%]}\end{array}$ & $\begin{array}{l}19 / 547(3.5 \%) \\
{[1.9 \%-5.0 \%]}\end{array}$ \\
\hline & High risk $(>4)$ & $\begin{array}{l}0 / 19(0 \%) \\
{[0 \%-0 \%]}\end{array}$ & $\begin{array}{l}3 / 80(3.7 \%) \\
{[0 \%-7.9 \%]}\end{array}$ & $\begin{array}{c}25 / 127(19.7 \%) \\
{[12.8 \%-26.6 \%]}\end{array}$ & $\begin{array}{l}28 / 226(12.4 \%) \\
{[8.1 \%-16.7 \%]}\end{array}$ \\
\hline & Total & $\begin{array}{l}0 / 70(0 \%) \\
{[0 \%-0 \%]}\end{array}$ & $\begin{array}{c}5 / 358(1.4 \%) \\
{[0.2 \%-2.6 \%]}\end{array}$ & $\begin{array}{l}42 / 345(12.2 \%) \\
{[8.7 \%-15.6 \%]}\end{array}$ & $\begin{array}{l}47 / 773(6.1 \%) \\
{[4.4 \%-7.8 \%]}\end{array}$ \\
\hline
\end{tabular}

high clinical risk would be expected to have a positive PE prevalence of $>10 \%$ on CTA. Patients with a negative or nonpositive d-dimer level with low clinical risk were noted to have a positive PE prevalence of $<2 \%$ and would not likely benefit from CTA.

\section{Discussion}

Pulmonary CTA has become the first-line imaging test for the diagnosis of acute PE [15]. The PIOPED II study demonstrated that CTA for pulmonary embolism had both good sensitivity (83\%) and specificity (96\%) [16].

However, pulmonary CTA has become over-utilized. The prevalence of PE in the patients included in the multicenter PIOPED II study was 23\% [16]. In the Dutch multicenter study utilizing CTA reported by the Christopher Study investigators, the prevalence of PE was $20 \%$ [9]. However, in three recent single institution retrospective studies, the prevalence of PE among subjects who underwent pulmonary CTA was much lower, ranging from $5.7 \%$ to $9.7 \%[2,13,17]$. The lower prevalence of $\mathrm{PE}$ in single center studies suggests that busy physicians going about their normal practice routine may not have the time to carefully assess patients as would occur prior to enrollment of subjects in a prospective multicenter trial. This is confirmed in the study by Adams et al., who found that among 3500 consecutive CTA studies performed at two urban emergency departments, only $45.5 \%$ of patients would have met the PIOPED recommendations for the evaluation of PE, while the majority did not have sufficiently high clinical risk or had a negative d- dimer value [18].

While there has been an increase in usage of CTA for the diagnosis of PE, there is little data to suggest that this has reduced patient morbidity or mortality. In fact, there is now debate whether pulmonary embolism carries as high of mortality as has previously been suggested [3]. In addition, all pulmonary emboli detected by CTA may not be clinically relevant, especially when they involve only the subsegmental pulmonary arterial branches [7].

In an era of escalating healthcare costs with insufficient means of funding, over-utilization of any expensive imaging examination requires close examination. CTA is especially relevant because in addition to cost, CTA studies carry inherent risks. An important concern for many patients, especially young women, undergoing evaluation for $\mathrm{PE}$ is patient exposure to ionizing radiation [4,5,19-21]. A review of literature from 1980-2007 found the average effective dose from a chest CT for PE in adults to be 15 $\mathrm{mSv}$ (ranging from $13-40 \mathrm{mSv}$ ) [21]. The proportion of cancers in the U.S. attributable to radiation exposure from CT studies has increased from an estimated $0.4 \%$ in 1991-1996, to currently approaching 2\% [4].

Contrast-induced nephropathy is another concern for many patients. In a prospective study, Mitchell et al. found the risk of contrast-induced nephropathy (CIN), defined as an increase in the serum creatinine level after control administration of $\geq 0.5 \mathrm{mg} / \mathrm{dL}$ or $\geq 25 \%$ from the baseline level, to be $14 \%$ among 174 consecutive patients evaluated for PE by contrast-enhanced CTA [22]. Furthermore, the development of contrast-induced neph- 
ropathy after CTA significantly increased the risk of the composite outcome of severe renal failure or death from renal failure within 45 days [22]. This risk does not appear to be mitigated by the use of different contrast agents. In a study specifically addressing the use of low-osmolar versus iso-osmolar nonionic contrast agents in patients with renal insufficiency, there was no statistically significant difference in the overall incidence of CIN [6].

Estimating the overall prevalence of acute $\mathrm{PE}$ in a patient population may be important prior to setting the appropriate thresholds of clinical probability and serum d-dimer values. If the overall prevalence of PE is low, then higher thresholds may be necessary to reduce false positive studies. Several studies have evaluated the prevalence of coincidental PE on CT studies of the chest performed for other indications. Farrell et al. found 10 cases of incidental PE among 440 outpatients undergoing contrast-enhanced chest CT for other indications, a prevalence of $2.2 \%$ [11]. Hui et al. found a $1.8 \%$ prevalence of coincidental PE among 1168 patients undergoing $\mathrm{CT}$ for other indications, most of whom (82.8\%) were outpatients [10]. In a meta-analysis including over 10,000 subjects, Dentali et al. found an overall prevalence of incidental PE on chest CT of 2.6\% [12]. From these data, we would argue that patients should have greater than $2 \%$ probability of $\mathrm{PE}$ in order to justify a CTA study.

Bayes theorem can be used to estimate the likelihood of a detecting a true positive versus false positive pulmonary embolism assuming pulmonary CTA has a sensitivity of $83 \%$ and specificity of $96 \%$ as reported in the PIOPED II study [16]. If the prevalence of PE within a population is only $2 \%$, then application of Bayes theorem would predict that approximately $70 \%$ of all positive CTA studies for pulmonary embolism would be incorrect, i.e., be falsely positive. At such a low prevalence, roughly 2 patients would be harmed with long-term anticoagulation in order to correctly treat 1 patient. Utilizing CTA for only patient groups with a PE prevalence of $10 \%$ as suggested by Costantino [1] seems more reasonable since application of the Bayes theorem to such a population would predict that $70 \%$ of positive CTA studies for pulmonary embolism would now be correct. At a $10 \%$ prevalence of $\mathrm{PE}$, the appropriate anticoagulation of every 2 patients would be offset by the inappropriate anticoagulation of 1 patient.

Two previous studies have demonstrated that in patients with negative or nonpositive serum d-dimer levels, there is a high likelihood of incorrect assessment of pulmonary embolism by CTA. Hirai-Gimber et al. found that among 7 subjects with a nonpositive serum d-dimer value and a positive pulmonary embolism initially reported on CTA, 6 were felt to be incorrect on both blinded and specialist review. All 6 subjects had low clinical risk and did not have ancillary imaging findings suspicious for PE [23]. In another study of subjects with negative serum d-dimers, both CTA studies initially reported to be positive for PE in the patient group were felt to be negative on specialist review [24]. This is an important consideration because of the significant risks associated with long-term anticoagulation. In a study of 2147 patients with venous thromboembolism treated with oral anticoagulation, 169 patients (7.9\%) suffered a major bleed requiring hospitalization at an average cost of $\$ 15,339 \pm$ $\$ 52,029$ (median, \$4999) per bleed, and 612 patients $(28.5 \%)$ suffered at least one bleed event that did not require hospitalization at an average cost of $\$ 239 \pm \$ 386$ (median, \$95) per event [25].

Setting a higher d-dimer value threshold appears to increase specificity without compromising sensitivity, thereby further reducing unnecessary CTA procedures. Both absolute and variable increased d-dimer thresholds have been proposed. Abcarian et al. noted that among 159 patients who had a low but positive d-dimer level of $\leq 1$ $\mathrm{ug} / \mathrm{ml}$ using an automated latex agglutination assay (a negative value is $\leq 0.4 \mathrm{ug} / \mathrm{mL}$ ), none had a positive pulmonary CTA study [13]. In a large prospective multicenter trial of 678 subjects evaluated for pulmonary embolism by pulmonary CTA, the authors used two different d-dimer thresholds using the VIDAS quantitative assay (VIDAS; Biome'rieux, Durham, NC, USA): the conventional $0.5 \mathrm{ug} / \mathrm{mL}$ threshold and a doubling to 1 $\mathrm{ug} / \mathrm{mL}$. In this study, CTA served as the diagnostic standard. For low risk subjects (Wells score $\leq 4$ ), 4 patients with serum d-dimer values $<0.5 \mathrm{ug} / \mathrm{mL}$ were noted by CTA to have PE. This number increased to 12 patients with the higher d-dimer threshold [26]. However, the authors noted that all of the 8 additional patients had pulmonary embolism limited to the subsegmental branches, making their clinical relevance uncertain as none of them had evidence of leg venous thrombosis [26]. Using the combination of low clinical risk and higher d-dimer threshold could have prevented a CTA study in $1 / 3$ of all patients. Kabrhel et al. observed that for a large population of patients at low clinical risk for pulmonary embolism (prevalence of $2.9 \%$ ), doubling the d-dimer threshold for any quantitive d-dimer assay resulted in a significant increase in specificity from $63 \%$ to $86 \%$ without a significant decrease in the negative predictive value (99.6\% to $98.9 \%)$ [27].

Our data demonstrates that patients in this study with a d-dimer $<1.0 \mathrm{ug} / \mathrm{ml}$ and a low Wells score have a prevalence of PE less than $2 \%$ and do not require $\mathrm{CT}$ imaging. We found that utilizing pulmonary CTA only in patients suspected of pulmonary embolism with a combination of intermediate or high clinical risk based on the Wells criteria (Wells score $\geq 2$ ) and a serum d-dimer cutoff of 1 $\mu \mathrm{g} / \mathrm{ml}$, would increase the prevalence of positive pulmo- 
nary CTA studies above 10\%. Furthermore, it appears that patients with a negative serum d-dimer value do not require further $\mathrm{CT}$ imaging for pulmonary embolism irrespective of their clinical risk stratification. We recommend the implementation of d-dimer measurement and Wells score evaluation prior to utilizing pulmonary CTA. If patients have serum d-dimer values above $1 \mu \mathrm{g} / \mathrm{ml}$ and clinical risk based on Wells criteria greater than 2 , then physician may order the CT study. Such a strategy would have avoided 422 (54\%) CTA studies while missing only 5 possible cases of pulmonary embolism, a prevalence less than $2 \%$.

A primary limitation of this study was the necessity to assign Wells scores retrospectively through electronic medical record review. Electronic medical record review may provide less information in comparison to direct patient examination. However, this limitation was decreased in this population because all belonged to an HMO in which all inpatient and outpatient data are recorded in a common electronic medical record. Using a single reviewer to assess the medical record using a standard protocol reduced the interobserver variability that can result from having multiple different physicians performing the evaluation. Another limitation is that a single population analysis may not provide necessary prevalence information to implement in all practices. Therefore, before these results can be applied in other medical centers, the underlying prevalence of PE in each center may need to be calculated. Interestingly, prevalence data from other published single center studies of patients evaluated for PE in the emergency department seem in line with our own $[1,2,17]$. Finally, this HMO utilizes only one method of serum d-dimer measurement (STA D-DI; DiagnosticaStago, Parsippany, NJ). Therefore, it is not clear whether the use of the $1.0 \mathrm{ug} / \mathrm{mL}$ threshold value could be applied to other methods of d-dimer measurement.

In conclusion, utilizing a clinical decision rule with an increased d-dimer threshold has several important advantages with associated large reduction in unnecessary CTA examinations and subsequent reduction in ionizing radiation exposure, less contrast induced nephropathy, lower likelihood of false positive CTA studies, decreased risk of unnecessary anticoagulation, and lower overall cost of healthcare.

\section{REFERENCES}

[1] M. M. Costantino, G. Randall, M. Gosselin, M. Brandt, K. Spinning and C. D. Vegas, "CT Angiography in the Evaluation of Acute Pulmonary Embolism," American Journal of Roentgenology, Vol. 191, No. 2, 2008, pp. 471-474. doi:10.2214/AJR.07.2552

[2] J. D. Prologo, R. C. Gilkeson, M. Diaz and J. Asaad, "CT Pulmonary Angiography: A Comparative Analysis of the
Utilization Patterns in Emergency Department and Hospitalized Patients between 1998 and 2003," American Journal of Roentgenology, Vol. 183, No. 4, 2004, pp. 1093-1096. doi:10.2214/ajr.183.4.1831093

[3] K. K. Calder, M. Herbert and S. O. Henderson, "The Mortality of Untreated Pulmonary Embolus in Emergency Department Patients," Annals of Emergency Medicine, Vol. 45, No. 3, 2005, pp. 302-310.

doi:10.1016/j.annemergmed.2004.10.001

[4] D. J. Brenner and E. J. Hall, "Computed TomographyAn Increasing Source of Radiation Exposure," Annals of Emergency Medicine, Vol. 357, No. 22, 2007, pp. 22772284. doi:10.1056/NEJMra072149

[5] A. Sodickson, P. F. Baeyens, K. P. Andriole, L. M. Prevedello, R. D. Nawfel, R. Hanson and R. Khorasani, "Recurrent CT, Cumulative Radiation Exposure, and Associated Radiation-Induced Cancer Risks from CT of Adults," Radiology, Vol. 251, No. 1, 2009, pp. 175-184. doi:10.1148/radiol.2511081296

[6] M. J. Kuhn, N. Chen, D. V. Sahani, D. Reimer, E. J. R. van Beek, J. P. Heiken and G. J. So, "The PREDICT Study: A Randomized Double-Blind Comparison of Contrast-Induced Nephropathy after Low- or Isoosmolar Contrast Agent Exposure," American Journal of Roentgenology, Vol. 191, No. 1, 2008, pp. 151-157. doi:10.2214/AJR.07.3370

[7] M. Carrier, M. Righini, P. S. Wells, A. Perrier, D. R. Anderson, M. A. Rodger, S. Pleasance and G. Le Gal, "Subsegmental Pulmonary Embolism Diagnosed by Computed Tomography: Incidence and Clinical Implications: A Systematic Review and Meta-Analysis of the Management Outcome Studies," Journal of Thrombosis and Haemostasis, Vol. 8, No. 8, 2010, pp. 1716-1722. doi:10.1111/j.1538-7836.2010.03938.x

[8] P. S. Wells, D. R. Anderson, M. Rodger, I. Stiell, J. F. Dreyer, D. Barnes, M. Forgie, G. Kovacs, J. Ward and M. J. Kovacs, "Excluding Pulmonary Embolism at the Bedside without Diagnostic Imaging: Management of Patients with Suspected Pulmonary Embolism Presenting to the Emergency Department by Using a Simple Clinical Model and D-Dimer," Journal of Thrombosis and Haemostasis, Vol. 135, 2001, pp. 98-107. doi:10.7326/0003-4819-135-2-200107170-00010

[9] Writing Group for the Christopher Study Investigators, "Effectiveness of Managing Suspected Pulmonary Embolism Using an Algorithm Combining Clinical Probability, D-Dimer Testing, and Computed Tomography," The Journal of the American Medical Association, Vol. 295, No. 2, 2006, pp. 172-179. doi:10.1001/jama.295.2.172

[10] G. C. Hui, A. Legasto and C. Wittram, "The Prevalence of Symptomatic and Coincidental Pulmonary Embolism on Computed Tomography," Journal of Computer Assisted Tomography, Vol. 32, No. 5, 2008, pp. 783-787. doi:10.1097/RCT.0b013e31815a7aea

[11] C. Farrell, M. Jones, F. Girvin, G. Ritchie, J. T. Murchison, "Unsuspected Pulmonary Embolism Identified Using Multidetector Computed Tomography in Hospital Outpatients," Clinical Radiology, Vol. 65, No. 1, 2010, pp. 1-5.

[12] F. Dentali, W. Ageno, C. Becattini, L. Galli, M. Gianni, N. Riva, D. Imberti, A. Squizzato, A. Venco and G. Ag- 
nelli, "Prevalence and Clinical History of Incidental, Asymptomatic Pulmonary Embolism: A Meta-Analysis," Thrombosis Research, Vol. 125, No. 6, 2010, pp. 518-522. doi:10.1016/i.thromres.2010.03.016

[13] P. W. Abcarian, J. D. Sweet, J. T. Watabe and H. -C. Yoon, "Role of a Quantitative D-Dimer Assay in Determining the Need for CT Angiography of Acute Pulmonary Embolism," American Journal of Roentgenology, Vol. 182, No. 6, 2004, pp. 1377-1381. doi:10.2214/ajr.182.6.1821377

[14] L. K. Hirai, J. M. Takahashi and H.-C. Yoon, "A Prospective Evaluation of a Quantitative D-Dimer Assay in the Evaluation of Acute Pulmonary Embolism," Journal of Vascular and Interventional Radiology, Vol. 18, No. 8, 2007, pp. 970-974. doi:10.1016/j.jvir.2007.04.020

[15] The Task Force for the Diagnosis and Management of Acute Pulmonary Embolism of the European Society of Cardiology (ESC), "Guidelines on the Diagnosis and Management of Acute Pulmonary Embolism," European Heart Journal, Vol. 29, No. 18, 2008, pp. 2276-2315. doi:10.1093/eurheartj/ehn310

[16] P. D. Stein, S. E. Fowler, L. R. Goodman, A. Gottschalk, C. A. Hales, R. D. Hull, K. V. Leeper, J. Popovich, D. A. Quinn, T. A. Sos, H. D. Sostman, V. F. Tapson, T. W. Wakefield, J. G. Weg and P. K. Woodard, "Multidetector computed tomography for acute pulmonary embolism," The New England Journal of Medicine, Vol. 354, No. 22, 2006, pp. 2317-2327. doi:10.1056/NEJMoa052367

[17] J. H. Donohoo, W. W. Mayo-Smith, J. A. Pezzullo and T. K. Egglin, "Utilization Patterns and Diagnostic Yield of 3421 Consecutive Multidetector Row Computed Tomography Pulmonary Angiograms in a Busy Emergency Department," Journal of Computer Assisted Tomography, Vol. 32, No. 3, 2008, pp. 421-425. doi:10.1097/RCT.0b013e31812e6af3

[18] D. M. Adams, S. M. Stevens, S. C. Woller, R. S. Evans, J. F. Lloyd, G. L. Snow, T. L. Allen, J. R. Bledsoe, L. M. Brown, D. P. Blagev, T. D. Lovelace, T. L. Shill, K. E. Conner, V. T. Aston and C. G. Elliott, "Adherence to PIOPED II Investigators' Recommendations for Computed Tomography Pulmonary Angiography," American Journal of Medicine, Vol. 126. No. 1, 2013, pp. 36-42. doi:10.1016/j.amjmed.2012.05.028

[19] M. V. Huppmann, W. B. Johnson and M. C. Javitt, "Radiation Risks from Exposure to Chest Computed Tomography," Seminars in Ultrasound, CT, and MRI, Vol. 31,
No. 1, 2010, pp. 14-28.

[20] J. Kuriakose and S. Patel, "Acute Pulmonary Embolism," Radiologic Clinics of North America, Vol. 48, No. 1, 2010, pp. 31-50.

[21] F. A. Mettler, W. Huda, T. T. Yoshizumi and M. Mahesh, "Effective Doses in Radiology and Diagnostic Nuclear Medicine: A Catalog," Radiology, Vol. 248, No. 1, 2008, pp. 254-263. doi:10.1148/radiol.2481071451

[22] A. M. Mitchell, A. E. Jones, J. A. Tumlin and J. A. Kline, "Prospective Study of the Incidence of Contrast-Induced Nephropathy among Patients Evaluated for Pulmonary Embolism by Contrast-Enhanced Computed Tomography," Academic Emergency Medicine, Vol. 19, No. 6, 2012, pp. 618-625. doi:10.1111/j.1553-2712.2012.01374.x

[23] L. Hirai-Gimber, R. I. Travis, J. M. Takahashi, T. L. Goodman and H.-C. Yoon, "Computed Tomography Angiography in Patients Evaluated for Acute Pulmonary Embolism with Low Serum D-Dimer Levels: A Prospective Study," The Permanente Journal, Vol. 13, No. 4, 2009, pp. 4-10.

[24] P. Deonarine, C. de Wet and A. McGhee, "Computed Tomographic Pulmonary Angiography and Pulmonary Embolism: Predictive Value of a D-Dimer Assay," BMC Research Notes, Vol. 5, No. 1, 2012, p. 104. doi:10.1186/1756-0500-5-104

[25] M. F. Bullano, V. Willey, O. Hauch, G. Wygantand, A. C. Spyropoulos and L. Hoffman, "Longitudinal Evaluation of Health Plan Cost Per Venous Thromboembolism or Bleed Event in Patients with a Prior Venous Thromboembolism Event During Hospitalization," Journal of Managed Care Pharmacy, Vol. 11, No. 8, 2005, pp. 663673.

[26] J. A. Kline, M. M. Hogg, D. M. Courtney, C. D. Miller, A. E. Jones and H. A. Smithline, "D-Dimer Threshold Increase with Pretest Probability Unlikely for Pulmonary Embolism to Decrease Unnecessary Computerized Tomographic Pulmonary Angiography," Journal of Thrombosis and Haemostasis, Vol. 10, No. 4, 2012, pp. 572-581. doi:10.1111/j.1538-7836.2012.04647.x

[27] C. Kabrhel, M. Courtney, C. A. CamargoJr, C. L. Moore, P. B. Richman, M. C. Plewa, K. E. Nordenholtz, H. A. Smithline, D. M. Beam, M. D. Brown and J. A. Kline, "Potential Impact of Adjusting the Threshold of the Quantitative D-Dimer Based on Pretest Probability of Acute Pulmonary Embolism," Academic Emergency Medicine, Vol. 16, No. 4, 2009, pp. 325-332. doi:10.1111/j.1553-2712.2009.00368.x 